\title{
The Effect of Beams' Orientations on the Intensity-Modulated Radiation Therapy Plan Quality
}

Ehab M Attalla* and Ismail Eldesoky

Children's Cancer Hospital, National Cancer Institute, Cairo University, Cairo, Egypt

${ }^{*}$ Corresponding author: Ehab M Attalla, Children's Cancer Hospital, Cancer Institute, Cairo University, Cairo, Egypt. Tel: +201005373359; E-mail: attalla.ehab@gmail.com

Received date: January 16, 2017; Accepted date: January 20, 2017; Published date: January 23, 2017

Copyright: ( 2017 Attalla EM, et al. This is an open-access article distributed under the terms of the Creative Commons Attribution License, which permits unrestricted use, distribution, and reproduction in any medium, provided the original author and source are credited.

\begin{abstract}
Purpose: The work aims at studying forty Intensity-modulated radiation therapy (IMRT) plans designed using KonRad system and evaluating each of them under different conditions to study the effect of beams' orientations on the plan quality.

Methods: Clinical step-and-shoot IMRT treatment plans were designed for twenty patients, suffering from different types of non-CNS solid tumors, to be delivery on a Siemens Oncor accelerator with multi-leaf collimators MLCs (82 leaf). To ensure that the similarity or difference between the plans was due to effect of beams' orientations, the same optimization constraints were applied for each plan and all other parameters were kept constant.
\end{abstract}

Results: The analysis of performance was based on isodose distributions, Dose Volume Histograms (DVHs) for Planning Target Volume (PTV), the relevant Organs at Risk (OARs) as well as several physical indices like mean dose $\left(D_{\text {mean }}\right)$, maximum dose $\left(D_{\text {max }}\right), 95 \%$ dose (D95), integral dose, volume of tumor receiving 2 Gy and 5 Gy, total number of segments and monitor units (MUs). Homogeneity index and conformation number were two other evaluation parameters that were considered in this study.

Conclusion: All coplanar CP and non-coplanar NC techniques result in clinically acceptable plans, with comparable target doses and dose to critical organs within prescribed dose constraints. For different organs at risk, the coplanar IMRT, the greatest advantage of non-coplanar IMRT was the marked reduction of general trend was that non-coplanar plans achieved the lowest values, while coplanar plans showed the highest. Significant differences $(P<0.05)$ exist only between the homogeneity index $\mathrm{HI}(1.092$ and 1.088) and Monitor Unit MU (357.79 and 341.80) of $\mathrm{CP}$ and NC plans respectively. Compared with the integral dose $(p=0.007)$.

Keywords: Beams' orientations; Intensity-modulated radiation therapy; Dose distribution; KonRad

\section{Introduction}

Radiation efficiency and treatment-plan quality are closely tied to beam angle arrangements and the number of beams used. Research into computer optimization of beam-angle selection has not proved to be clinically useful because of the large angular search space and the dependence on a specific clinical situation [1].

Wang et al. [2] studied the effectiveness of noncoplanar beam configurations and the benefit of plans using few but optimally placed beams and treatment plans optimized using the parallelized multipleresolution beam angle optimization (PMBAO) approach were at least equivalent to, and overall better than, the plans using common equally spaced coplanar beams. The observation that coplanar techniques irradiate OARs to adequately treat adjacent PTV led to the hypothesis that techniques that involve noncoplanar beam arrangements may decrease doses to the critical structures. While non-coplanar IMRT plan usually takes longer treatment planning time and it requires complex patient setup and treatment [3].
Raymond et al. [4] compared dosimetric and radiobiological parameters of treatment plans using coplanar and noncoplanar beam arrangements in patients with fronto-temporal high-grade glioma generated for IMRT or volumetric-modulated arc therapy (VMAT). They concluded that technique selection should be individualized, based on patient-specific clinical and dosimetric parameters. Chang et al. [5] examined the utility of noncoplanar beam angles in IMRT for the management of 16 pancreatic cancer patients. The use of NCP beam angles was preferred only in patients with risk factors for treatment-related kidney dysfunction. The purpose of this study was to compare dosimetric parameters of treatment plans using coplanar and noncoplanar beam arrangements in patients with different selected cases generated for intensity-modulated radiotherapy (IMRT).

\section{Materials}

This is a retrospective study Case selection was limited to patients in whom the planning target volume (PTV) overlapped or was located within $3 \mathrm{~mm}$ of one of OARs. Twenty patients were enrolled in the retrospective study Table 1 . All patients were immobilized in supine position with a customized cushion and thermoplastic masks followed by computed tomography (CT) scanning. The CT slices were acquired every $2 \mathrm{~mm}$; The CT images were then transferred to the TPS via local 
Page 2 of 6

area network. The photon beam energy for all plans was $6 \mathrm{MV}$ delivered from Siemens linac equipped with 41 pair-leaf MLC. A consensus set of structures was delineated according to department's clinical protocol, which was similar to the guideline of RTOG, by radiation oncologist for the target, OARs and treatment volumes. The following organs at risk were contoured and included in the analysis: Brain, Brainstem, optic chiasm, RT Cochlea, LT Cochlea, both eyes and lenses.

\begin{tabular}{|c|c|c|c|}
\hline Diagnosis & Prescribed Dose (Gy) & PTV Volume (CC) & Patient Volume (CC) \\
\hline Ependymoma & 54 & 162 & 2569 \\
\hline Ependymoma & 59.4 & 254 & 4371 \\
\hline Ependymoma & 54 & 186 & 2171 \\
\hline Ependymoma (TB) & 59.4 & 226 & 2537 \\
\hline PF Eependymoma & 54 & 189 & 10089 \\
\hline Meningioma & 60 & 759 & 3867 \\
\hline Astrocytoma & 59.4 & 112 & 3112 \\
\hline High Grade Glioma & 54 & 189 & 2821 \\
\hline Base of Skull Chadama & 64.8 & 15 & 4143 \\
\hline Craniopharyngioma & 54 & 43 & 4311 \\
\hline Craniopharyngioma & 54 & 64 & 3176 \\
\hline Craniopharyngioma & 54 & 27 & 3503 \\
\hline Craniopharyngioma & 54 & 65 & 2988 \\
\hline Craniopharyngioma & 54 & 162 & 4661 \\
\hline Retinoblastoma & 41.4 & 30 & 1314 \\
\hline Retinoblastoma & 41.4 & 42 & 2064 \\
\hline LT eye & 45 & 29 & 2795 \\
\hline LT nasal & 50.4 & 87 & 2297 \\
\hline $\mathrm{NPH}$ & 60 & 108 & 4507 \\
\hline LT ingyuinal & 59.4 & 534 & 10729 \\
\hline
\end{tabular}

Table 1: The Diagnosis, prescription dose, PTV Volume and Patient Volume for the cases used to study the effect of beam orientation on the quality of IMRT plans.

\section{Treatment protocol and plan evaluation}

Both coplanar and no coplanar plans were generated for each of the selected cases. Planning was performed by an experienced dosimetrist. Optimization was performed on the study inverse planning system konrad using the Pencil beam dose-calculation algorithm [6]. The planning goal was to have the minimum volume of the PTV covered by the $95 \%$ isodose line was $95 \%$. The maximum dose permitted within the PTV was $110 \%$.

Planning commenced with conventional coplanar IMRT using approximately equally-spaced entrance and exit gantry angles. These plans consisted of 9 fields for midline PTVs and 7 fields for more lateral volumes. Fields were applied to the patients with angles $0^{\circ}, 40^{\circ}$, $80^{\circ}, 120^{\circ}, 160^{\circ}, 200^{\circ}, 240^{\circ}, 280^{\circ}$, and $320^{\circ}$ for Coplanar IMRT plans while Noncoplanar IMRT plans consist of 7 beams in the same plane and two in different plane usually 30,330 or 90 with couch angle 270 . Couch rotations were limited to one or two angles. In addition, to limit the irradiation by noncoplanar beams of normal tissue located superiorly and inferiorly outside the PTV, we contoured blocking structures at the entry point of the two noncoplanar beams, outside the superior and inferior borders of the PTV. Each plan was evaluated with respect to isodose distributions and dose-volume histograms (DVHs), which were calculated for all the delineated volumes in the two different treatment plans. Additional dosimetric parameters related to the doses received by the PTV included mean, maximum PTV doses and OARs were compared quantitatively. It also included homogeneity index and conformity index as defined by Riet et al. [7] where $\mathrm{CN}=\mathrm{TV}_{\mathrm{RI}} / \mathrm{TV}^{\star} \mathrm{TV}_{\mathrm{RI}} / \mathrm{V}_{\mathrm{RI}} ; \mathrm{CN}=$ Conformation Number, $\mathrm{TV}_{\mathrm{RI}}=$ Target Volume covered by the Reference Isodose, TV=Target Volume and $\mathrm{V}_{\mathrm{RI}}=$ volume of the reference isodose.

According to the International Commission on Radiation Units and measurements ICRU the reference isodose is $95 \%$. Homogeneity index HI was defined as the difference between the doses covering $5 \%$ and 95\% of the PTV. The equation is as follows: $\mathrm{HI}=\mathrm{D}_{5 \%} / \mathrm{D}_{95 \%}$. A greater value of $\mathrm{HI}$ indicates a greater degree of dose heterogeneity in the PTV. 
Citation: Attalla EM, Eldesoky I (2017) The Effect of Beams' Orientations on the Intensity-Modulated Radiation Therapy Plan Quality. J Nucl Med

Page 3 of 6

For the PTV, the parameters recommended in ICRU Report No. 83 were adopted; these were $\mathrm{D}_{98 \%}$ (dose delivered to $98 \%$ of the volume of the PTV), $\mathrm{D}_{2 \%}$ (dose delivered to $2 \%$ of the volume of the PTV), mean dose and dose standard deviation. The different plans were compared using mean statistics. The differences between means were tested for significance using a two-tailed paired Student's t-test. The null hypothesis was that there was no difference between the coplanar IMRT technique and the non-coplanar IMRT treatment techniques. Statistical significance was set at $\mathrm{P}<0.05$.

\section{Results and Discussion}

\section{Conformity and homogeneity of the target}

Figure 1 shows the DVH's and the dose distributions for the coplanar IMRT technique (CP) and the non-coplanar IMRT technique (NC) for some of the investigated cases. DVH's for planning target volumes (PTVs) and the dose delivered to OARs are analyzed. The target coverage parameters are given in Table 2.
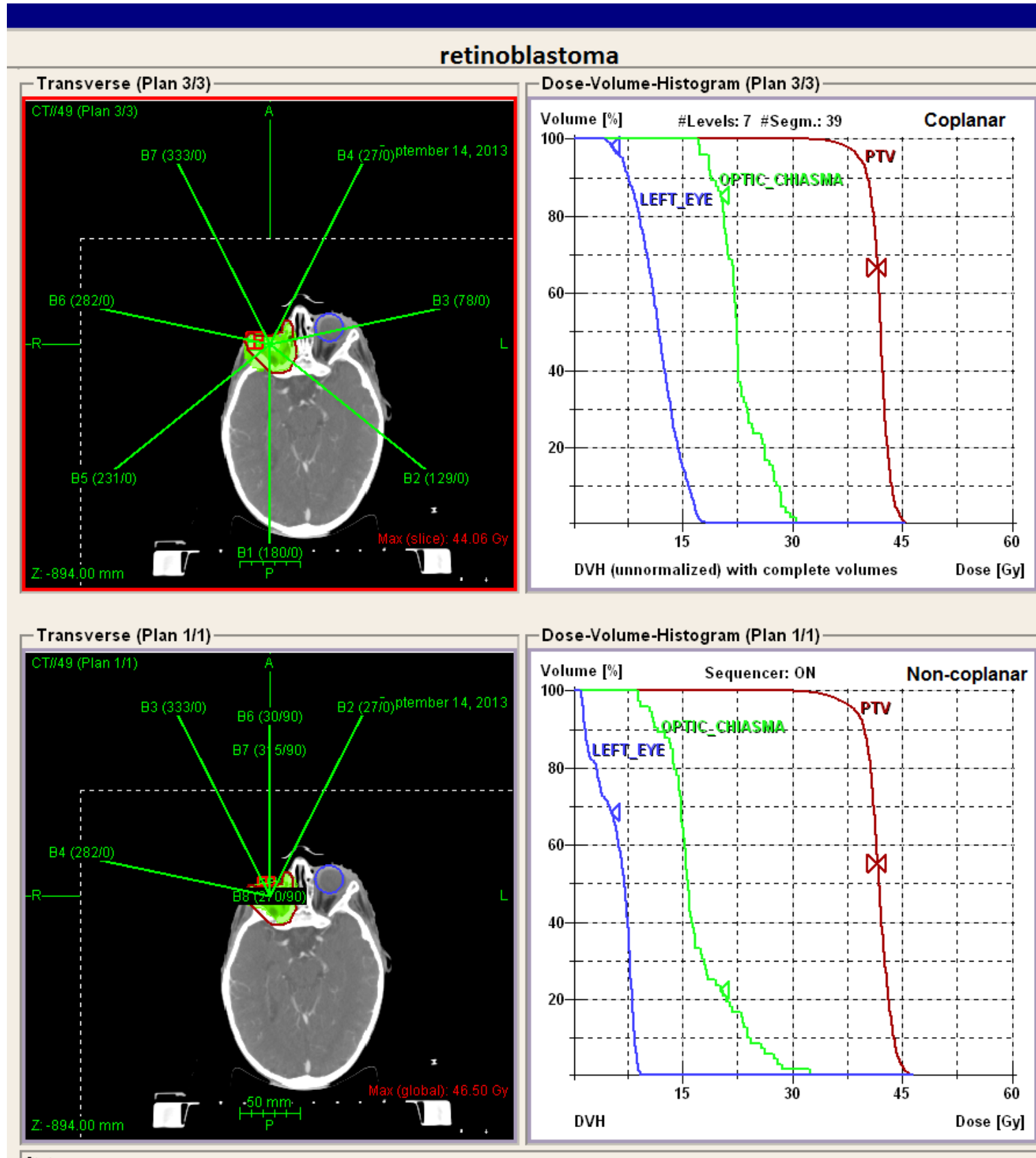

Status

Figure 1: The dose distribution and DVH's for the coplanar IMRT technique and the non-coplanar IMRT technique for retinoblastoma case. 
Citation: Attalla EM, Eldesoky I (2017) The Effect of Beams' Orientations on the Intensity-Modulated Radiation Therapy Plan Quality. J Nucl Med

Page 4 of 6

The two techniques result in comparable $\mathrm{D}_{\text {mean }}, \mathrm{D}_{\max }, \mathrm{D}_{98 \%}, \mathrm{D}_{95 \%}$, $\mathrm{D}_{5 \%}, \mathrm{~V}_{95 \%}$ and $\mathrm{V}_{107 \%}$ for PTV coverage. The non-coplanar IMRT and coplanar IMRT plans are similar $(\mathrm{p}>0.05)$. Among the homogeneity index $(\mathrm{HI})$, conformation number $(\mathrm{CN})$, monitor units $(\mathrm{MU})$ and number of segments parameters (Table 3 ), significant differences $(\mathrm{P}<0.05)$ exist only between the HI $(1.092$ and 1.088$)$ and $\mathrm{MU}(357.79$ and 341.80) of CP and NC plans respectively.

\begin{tabular}{|c|c|c|c|c|c|c|c|c|}
\hline \multirow[t]{2}{*}{ Parameter } & \multicolumn{2}{|l|}{$\mathrm{D}_{\max }(\mathrm{Gy})$} & \multicolumn{2}{|l|}{$\mathbf{D}_{\text {mean }}(\mathbf{G y})$} & \multicolumn{2}{|l|}{$\mathrm{D}_{95 \%}(\%)$} & \multicolumn{2}{|l|}{$\mathrm{D}_{98 \%}(\%)$} \\
\hline & CP & NC & CP & NC & CP & NC & CP & NC \\
\hline Mean \pm SD & $59.0 \pm 7.1$ & $58.6 \pm 6.5$ & $54.5 \pm 6.2$ & $54.5 \pm 6.2$ & $95.6 \pm 1.2$ & $95.5 \pm 1.5$ & $93.4 \pm 1.8$ & $93.1 \pm 2.5$ \\
\hline P-Value & \multicolumn{2}{|r|}{0.09} & \multicolumn{2}{|c|}{0.58} & \multicolumn{2}{|c|}{0.68} & \multicolumn{2}{|c|}{0.47} \\
\hline \multirow[t]{2}{*}{ Parameter } & \multicolumn{2}{|c|}{$\mathrm{D}_{5 \%}(\%)$} & \multicolumn{2}{|c|}{$V_{95 \%}(\%)$} & \multicolumn{2}{|c|}{$\mathrm{V}_{107 \%}(\%)$} & & \\
\hline & $\mathrm{CP}$ & NC & $\mathrm{CP}$ & NC & $\mathrm{CP}$ & NC & & \\
\hline Mean \pm SD & $104.2 \pm 0.9$ & $104.3 \pm 1.4$ & $96.5 \pm 1.4$ & $96.2 \pm 1.7$ & $1.0 \pm 1.2$ & $1.1 \pm 1.9$ & & \\
\hline P-Value & \multicolumn{2}{|r|}{0.87} & \multicolumn{2}{|c|}{0.23} & \multicolumn{2}{|c|}{0.88} & & \\
\hline
\end{tabular}

Table 2: $\mathrm{D}_{\max }(\mathrm{Gy}), \mathrm{D}_{\text {mean }}(\mathrm{Gy}), \mathrm{D}_{95 \%}(\%), \mathrm{D}_{98 \%}(\%), \mathrm{D}_{5 \%}(\%), \mathrm{V}_{95 \%}(\%)$ and $\mathrm{V}_{107 \%}(\%)$ for PTVs in the non-coplanar (NC) IMRT and coplanar (CP)IMRT plans, where Dmax: maximum dose to organ, $\mathrm{D}_{\text {mean }}$ : mean dose to organ, $\mathrm{D}_{\mathrm{n} \%}$ : the percentage dose received by the $\mathrm{n} \%$ volume of the target volume, $\mathrm{V}_{\mathrm{n} \%}$ : the percentage volume irradiated by $\mathrm{n} \%$ of the prescribed dose.

\begin{tabular}{|c|c|c|c|c|c|c|c|c|}
\hline \multirow[t]{2}{*}{ Parameter } & \multicolumn{2}{|l|}{ HI } & \multicolumn{2}{|l|}{$\mathrm{CN}$} & \multicolumn{2}{|l|}{ MU } & \multicolumn{2}{|l|}{ Segments } \\
\hline & $\mathrm{CP}$ & NC & $\mathrm{CP}$ & NC & $\mathrm{CP}$ & $\mathrm{NC}$ & $\mathrm{CP}$ & $\mathrm{NC}$ \\
\hline Mean \pm SD & $1.09 \pm 0.02$ & $1.1 \pm 0.02$ & $0.68 \pm 0.10$ & $0.67 \pm 0.10$ & $357.8 \pm 84.9$ & $341.8 \pm 77.2$ & $57.7 \pm 21.4$ & $55.8 \pm 23.2$ \\
\hline P-Value & \multicolumn{2}{|c|}{0.03} & \multicolumn{2}{|c|}{0.14} & \multicolumn{2}{|c|}{0.02} & \multicolumn{2}{|c|}{0.14} \\
\hline
\end{tabular}

Table 3: The homogeneity index (HI), conformation number (CN), monitor units (MU) and number of segments in both of the coplanar (CP) and non-coplanar (NC) plans.

\section{Dose sparing of the OARs}

Table 4 presents a number of DVH parameters calculated for CP and NC plans of different OARs. The only significant $(\mathrm{P}<0.05)$ differences between $\mathrm{CP}$ and NC plans are reported for the Dmean (Gy) parameter of both eyes, where the NC plans (10.54 and 9.35) are always better than CP plans (12.66 and 11.97) for RT and LT eyes respectively.

\begin{tabular}{|l|l|l|l|l|}
\hline \multirow{2}{*}{ OARs } & DVH parameter & CP & NC & P-value \\
\hline & $V_{5 G y}(\%)$ & $73.1 \pm 20.6$ & $69.5 \pm 13.9$ & 0.21 \\
\cline { 2 - 5 } & $V_{10 G y}(\%)$ & $60.2 \pm 23.9$ & $60.0 \pm 16.7$ & 0.96 \\
\cline { 2 - 5 } & $V_{15 G y}(\%)$ & $49.1 \pm 23.1$ & $50.3 \pm 20.1$ & 0.12 \\
\hline LT Cochlea & $D_{\text {mean }}(G y)$ & $25.5 \pm 11.0$ & $25.0 \pm 10.6$ & 0.19 \\
\hline RT Cochlea & $D_{\text {mean }}(G y)$ & $23.1 \pm 10.7$ & $23.0 \pm 9.3$ & 0.29 \\
\hline Brain stem & Max $1 \mathrm{~cm}^{3}$ & $49.4 \pm 10.9$ & $49.3 \pm 11.6$ & 0.92 \\
\hline Optic chaism & Max $(G y)$ & $30.5 \pm 13.0$ & $29.9 \pm 14.5$ & 0.52 \\
\hline RT eye & $D_{\text {mean }}(G y)$ & $12.7 \pm 6.4$ & $10.5 \pm 6.3$ & $\mathbf{0 . 0 0 0 1}$ \\
\hline LT eye & $D_{\text {mean }}(G y)$ & $11.9 \pm 5.7$ & $9.4 \pm 6.4$ & $\mathbf{0 . 0 0 1}$ \\
\hline RT lens & $D_{\text {mean }}(G y)$ & $6.5 \pm 2.8$ & $5.1 \pm 2.9$ & 0.14 \\
\hline
\end{tabular}


Page 5 of 6

\begin{tabular}{|l|l|l|l|l|}
\hline LT lens & $\mathrm{D}_{\text {mean }}$ (Gy) & $5.6 \pm 2.1$ & $4.3 \pm 2.9$ & 0.23 \\
\hline
\end{tabular}

Table 4: The dose-volume parameters for different OARs for coplanar $(\mathrm{CP})$ and non-coplanar $(\mathrm{NC})$ plans, where $\mathrm{D}_{\text {mean }}$ : mean dose to organ, $\mathrm{V}_{\mathrm{nGy}}$ : the percentage volume irradiated by $\mathrm{n}$ Gy or more of a certain structure.

\section{Integral dose (ID) and dose to normal tissue}

Table 5 shows that for the integral dose, $D_{1 \%}(G y), D_{2 \%}(G y)$, and $\mathrm{D}_{5 \%}(\mathrm{~Gy})$, the CP plans significantly $(\mathrm{p}<0.05)$ reduce the dose to normal tissue (41.65, 55.50, 53.19 and 42.72 respectively) compared to NC plans (43.70, 55.98, 54.85 and 48.95 respectively).

\begin{tabular}{|c|c|c|c|c|c|c|}
\hline \multirow[t]{2}{*}{ Parameter } & \multicolumn{2}{|l|}{ Integral Dose } & \multicolumn{2}{|l|}{$\mathbf{D}_{1 \%}(\mathbf{G y})$} & \multicolumn{2}{|l|}{$\mathbf{D}_{2 \%}(\mathrm{~Gy})$} \\
\hline & $\mathrm{CP}$ & NC & $\mathrm{CP}$ & NC & $\mathrm{CP}$ & NC \\
\hline Mean & 41.7 & 43.7 & 55.5 & 55.9 & 53.2 & 54.9 \\
\hline SD & 30.7 & 31 & 6.4 & 6.4 & 7.1 & 6.5 \\
\hline$P$-value & 0.007 & & 0.009 & & 0.022 & \\
\hline \multirow[t]{2}{*}{ Parameter } & \multicolumn{2}{|c|}{$\mathrm{D}_{5 \%}$ (Gy) } & \multicolumn{2}{|c|}{$V_{2 G y}(\%)$} & \multicolumn{2}{|c|}{$\mathrm{V}_{5 \mathrm{~Gy}}(\%)$} \\
\hline & $\mathrm{CP}$ & $\mathrm{NC}$ & $\mathrm{CP}$ & NC & $\mathrm{CP}$ & NC \\
\hline Mean & 42.7 & 48.9 & 72.1 & 74.9 & 58.4 & 61.5 \\
\hline SD & 14.6 & 10.6 & 17.1 & 15.5 & 19.1 & 14.2 \\
\hline P-value & 0.001 & & 0.4 & & 0.31 & \\
\hline
\end{tabular}

Table 5: Integral dose and low dose distribution in healthy tissue, where $\mathrm{D}_{\mathrm{n} \%}$ : the dose received by the $\mathrm{n} \%$ volume of the organ volume, $\mathrm{V}_{\mathrm{nGy}}$ : the percentage volume irradiated by $n$ Gy or more of a healthy tissue.

Non-coplanar IMRT does have some disadvantages. One should bear in mind the remark reported by Kan et al. [8] who stated that the use of non-coplanar beams might increase the peripheral dose. Moreover, the non-coplanar beam setup requires changing the couch angle, which increases the overall treatment time substantially. This can be compensated by the significant reduction in the combination of the number of MUs and the number of segments which results in shorter delivery times. Since cancer patients may have difficulties lying on the treatment couch for long periods of time during the radiation delivery, shortening the IMRT treatment time decreases the risk that patients involuntarily move during radiation therapy. It also minimizes the risk of decreased tumor cell killing potentially associated with delivery times in the range of $15 \mathrm{~min}$ to $45 \mathrm{~min}[9,10]$.

\section{Conclusion}

The hypothesis of the study, focus on the importance of the beam orientation since such selected beam orientations can reduce overall time, making the IMRT more practically in the clinical implementation. In case of the Non-Coplanar beams; orientations provide an additional degree of freedom for IMRT treatment optimization and facilitate to improve the quality for some complicated plans.

Also, the study provides a realistic methodology to research the characteristics of the IMRT dose distributions as a function of beam angle and to design practical filtering beam angle optimization strategies for IMRT planning. Although a slight impact is shown on some of the OARs, both non-coplanar and coplanar techniques achieved comparable target coverage. The main advantage of coplanar IMRT is the marked reduction of the integral dose. Also the overall treatment time is reduced because the couch angle has not to be changed.

\section{References}

1. Takamiya R, Missett B, Weinberg V, Akazawa C, Akazawa P, et al. (2007) Simplifying intensity-modulated radiotherapy plans with fewer beam angles for the treatment of oropharyngeal carcinoma. J Appl Clin Med Phys 2: 26-36.

2. Wang X, Zhang X, Dong L, Liu H, Gillin M, et al. (2005) Effectiveness of noncoplanar IMRT planning using a parallelized multiresolution beam angle optimization method for paranasal sinus carcinoma. Int J Radiat Oncol Biol Phys 2: 594-601.

3. Stein J, Mohan R, Wang XH, Bortfeld T, Wu X, et al. (1997) Number and orientations of beams in intensity-modulated radiation treatments. Med Phys 24: 149-160.

4. Raymond PV, Ansbacher W, Zavgorodni S, Bendorffe B, Nichol A, et al. (2012) Coplanar versus noncoplanar intensity-modulated radiation therapy (IMRT) and volumetric-modulated arc therapy (VMAT) treatment planning for fronto-temporal high-grade glioma. J Appl Clin Med Phys 13: 3826-3829.

5. Chang DS, Bartlett GK, Das IJ, Cardenes HR (2013) Beam angle selection for intensity-modulated radiotherapy (IMRT) treatment of unresectable 
Citation: Attalla EM, Eldesoky I (2017) The Effect of Beams' Orientations on the Intensity-Modulated Radiation Therapy Plan Quality. J Nucl Med Radiat Ther 8: 324. doi:10.4172/2155-9619.1000324

Page 6 of 6

pancreatic cancer: are noncoplanar beam angles necessary? Clin Trans Oncol 9: 720-724.

6. Knöös T, Ceberg C, Weber L, Nilsson P (1994) Dosimetric verification of a pencil beam based treatment planning system. Phys Med Biol 39: 1609-1628.

7. Van't Riet A, Mak AC, Moerland MA, Elders LH, van der Zee W (1997) A conformation number to quantify the degree of conformality in brachytherapy and external beam irradiation: Application to the prostate. Int J Radiat Oncol Biol Phys 37: 731-736.
8. Kan MW, Leung LH, Kwong DL, Wong W, Lam N (2010) Peripheral doses from noncoplanar IMRT for pediatric radiation therapy. Med Dosim 4: 255-263.

9. Crooks SM, McAven LF, Robinson DF, Xing L (2002) Minimizing delivery time and monitor units in static IMRT by leaf-sequencing. Phys Med Biol 47: 3105-3116.

10. Verhey LJ (1999) Comparison of three-dimensional conformal radiation therapy and intensity-modulated radiation therapy systems. Semin Radiat Oncol 9: 78-98. 